\title{
RASGOS FONOPRAGMÁTICOS DEL ESPAÑOL MEXICANO Y LA ATENUACIÓN EN LOS PROCESOS COMUNICATIVOS DEL FRANCÉS
}

\section{PHONOPRAGMATIC FEATURES OF MEXICAN SPANISH AND MITIGATION IN THE COMMUNICATIVE PROCESSES OF FRENCH}

\author{
Orlando Valdez Vega \\ María Eugenia Flores Treviño \\ Facultad de Filosofía y Letras de la Universidad Autónoma de Nuevo León* \\ orlando.valdezvg@uanl.edu.mx \\ maria.florestr@uanl.edu.mx
}

Recibido: 08/02/2017

Aceptado: 07/04/2017

\begin{abstract}
Resumen
En esta investigación se realiza un estudio de los rasgos fonopragmáticos (Hidalgo, 2009) de la lengua francesa, en hispanohablantes del noreste mexicano para revisar las variaciones atenuadoras en función del género y de la cultura del hablante que introducen un cambio de sentido en ese sistema de lengua. Intravaia (2000) afirma que los elementos rítmico-melódicos juegan en todas las lenguas un rol semántico y que cada lengua posee sus propios rasgos prosódicos que permiten expresar toda clase de estados psíquicos como satisfacción, desprecio, decepción, enojo, entre otros. Por
\end{abstract}

\begin{abstract}
The present paper is a study of phonopragmatics features (Hidalgo, 2009) of the French language in Spanish-speaking people of the north-east Mexico. Our aim is to explore variation regarding the use of variation taking into account gender and culture of the different speakers. Intravia (2000) affirms that the rhythm-melodic elements play in every language a semantic role, and that each language possesses its own prosodic features that allow people to express all kinds of psychic states such as satisfaction, scorn, anger, and others. To conclude, we highlight the importance and
\end{abstract}

* Miembros del CA-UANL 245 consolidado "Lenguajes, discursos, semióticas. Estudios de la cultura en la región", en el proyecto "Pragmática: Traducción y Competencia Comunicativa", dentro del macroproyecto "La atenuación pragmática en su variación genérica: géneros discursivos orales y escritos en el español de España y América (Proyecto MINECO: FFI2016-75249P)".

Para citar este artículo / To cite this article: Valdez Vega, Orlando y Flores Treviño, María Eugenia (2018): Rasgos fonopragmáticos del español mexicano y la atenuación en los procesos comunicativos del francés. García Ramón, Amparo y Soler Bonafont, María Amparo (Eds.): ELUA: Estudios de atenuación en el discurso, Anexo IV, págs. 323-339.

Enlace / Link: http://dx.doi.org/10.14198/ELUA2018.Anexo4.18 
último, subrayamos la importancia e implicaciones de su enseñanza durante el aprendizaje de la lengua francesa.

PALABRAS CLAVE: prosodia, atenuación, fonética, fonología, interferencia. implications of the study for teaching French as a foreign language.

KEY WORDS: prosody, mitigation, phonetics, phonology, interference.

\section{INTRODUCCIÓN}

Aprender una lengua no es tarea sencilla, sobre todo si este aprendizaje se entiende en su dimensión globalizadora, con respecto a la adquisición de la competencia comunicativa. Yang (2015) afirma que escuchar, hablar, leer y escribir una lengua, son habilidades que se encuentran en estrecha correlación en el aprendizaje de una lengua extranjera ya que cada una de ellas favorece el aprendizaje de las demás y todas ellas en su conjunto benefician al desarrollo de la competencia comunicativa. Por esta razón es entendible que se fomente el dominio de cada una de estas habilidades durante el aprendizaje de la lengua meta. En este trabajo, se considera que no hay que dejar de lado en el aprendizaje y enseñanza de una lengua extranjera el desarrollo de la destreza fonético-fonológica, sobre todo al considerar que cada sistema de lengua posee sus propios rasgos, referidos a los matices semánticos en los mecanismos fónicos portadores de significado. De esta manera, poseer un buen dominio de la competencia fonético-fonológica es deseable, especialmente para los que se dedicarán a la enseñanza de lenguas extranjeras como es el caso de los sujetos que participaron en este estudio.

En este artículo los autores se enfocarán en el estudio de los componentes de la competencia fonológica descritos en el Marco Común Europeo de Referencia (MCER) para las lenguas (Conseil de l'Europe 2001) para exponer su función lingüística y supralingüística (Intravaia 2000), así como su trascendencia en los procesos comunicativos en una lengua extranjera para entender la importancia de su enseñanza (Valdez 2013). Puesto que el éxito en la comunicación depende de una actividad lingüística y supralingüística en su conjunto (Intravaia 2000) se analizará la importancia de cada una de ellas y se describirán algunos recursos usuales para la atenuación y la intensificación (Briz 2007) en los procesos comunicativos de la lengua francesa.

\section{ENSEÑANZA DE LA COMPETENCIA FONOLÓGICA}

Trajkova (2014) subraya precisamente la importancia de la formación inicial en fonética correctiva a los futuros docentes de lengua debido al impacto positivo o negativo que se tendrá en la enseñanza de francés como lengua extranjera (FLE) en las aulas. De acuerdo con el autor, un docente que no tuvo una formación en fonética correctiva será incapaz de visualizar la importancia de desarrollar esta competencia en sus futuros alumnos.

Coincidimos con Herrero y Andión (2012) quienes hacen notar que, frecuentemente, en los manuales de aprendizaje de lenguas extranjeras se integra sólo el apartado de fonética en los niveles de inicio y se descuida el desarrollo de esta competencia en los niveles avanzados. Esta orientación pedagógica no es suficiente frente a las evidentes dificultades fonéticofonológicas que persisten a menudo en los aprendientes a nivel de las inadecuaciones en la configuración de fonemas y de los rasgos prosódicos. 
Otro aspecto que Herrero y Andión (2012) resaltan es que la mayoría del material didáctico para la enseñanza de la pronunciación y de los ejercicios de fonética previstos en los métodos para la enseñanza de lenguas extranjeras no han sido concebidos para un grupo lingüístico específico; este es un ámbito que no debería descuidarse considerando que cada lengua posee su propio sistema fonético-fonológico y las dificultades para aprender el sistema de la lengua meta variarán en función del grado de semejanza que se tenga con el de la lengua materna.

La necesidad de desarrollar la competencia fónica se debe a que cada sistema de lengua posee un número determinado de sonidos que denominamos fonemas y que son necesarios para la comunicación. Vaissière (2011) define el fonema como la unidad más pequeña funcional de un sistema fonológico. Por su parte, Rolland (2011) explica la fonética como aquella que describe la manera en que están constituidos los sonidos de una lengua y cuáles son sus características acústicas.

Blanco y Nogueroles (2014) proponen la competencia fonético-fonológica, primeramente, como la habilidad de percibir y producir fonemas, así como sus diferentes realizaciones (alófonos). Además, permite detectar rasgos fonéticos como la labialidad, la sonoridad, la nasalidad u oclusión. Asimismo, esta competencia implica el dominio de la composición fonética de las palabras a nivel de su estructura silábica o de su secuencia acentual. Por último, involucra rasgos ligados al contexto fonológico como la elisión, la relajación articulatoria o la reducción de vocales atonas, así como el buen dominio del acento, la entonación y el ritmo de las oraciones.

Esta definición de Blanco y Nogueroles (2014) nos lleva a afirmar que, si bien es cierto que la fonética es imprescindible en el estudio científico de una lengua al encargarse de la exploración de los sonidos del lenguaje en su emisión y su recepción (Chiss, Filliolet y Maingueneau 2013), no bastaría dicho conocimiento intelectual y práctico para la plena adquisición de la competencia fonético-fonológica puesto que los rasgos fonológicos son también parte esencial en la comunicación (Conseil de l'Europe 2001).

Dada la importancia de desarrollar esta competencia en los aprendientes de lenguas extranjeras se entiende que es urgente y necesario llevar a cabo investigaciones al respecto, para descubrir las dificultades fónicas que experimentan numerosos aprendientes procedentes de diversos grupos lingüísticos y coadyuvar así en el desarrollo de esta competencia en los procesos de enseñanza-aprendizaje para grupos lingüísticos específicos.

\subsection{Interferencia en la comprensión-comunicación en lengua francesa}

La eficiente comunicación en lengua extranjera presenta diversas problemáticas complejas, entre ellas la interferencia. Para explicar este fenómeno, Orduz (2012) comenta que los aprendientes, al tener un nivel de lengua bajo, no logran identificar los sonidos específicos de la lengua meta, situación que los lleva a utilizar o crear un sonido nuevo cercano al real existente en lengua meta. De cierta manera, el hablante, a partir de su sistema fonológico de la lengua materna crea uno diferente con base en las reglas fonológicas de su propia lengua. Frente a esta problemática se entiende que buscar el desarrollo de la competencia fonéticofonológica durante el aprendizaje de una segunda lengua o extranjera se vuelve primordial.

Por otra parte, Anabalón y Vivanco (2014) respecto a la influencia de la lengua materna sobre L2 distinguen entre una transferencia negativa o interferencia y una transferencia posi- 
tiva o facilitadora. Cuando en la lengua meta se usa un elemento de la lengua materna y ese elemento no hace parte del sistema de la lengua extranjera se considera como un uso interferencial provocando una enunciación errónea o equivocada. Pero cuando dicho elemento sí hace parte de la lengua meta, se convierte entonces en una transferencia facilitadora o positiva.

Ahora bien, cuando se aprende una lengua extranjera aparecen errores o equivocaciones en la pronunciación del aprendiente debido a que, a menudo, se usa la lengua meta con la influencia del sistema fonológico de la lengua materna, el cual no forzosamente comunica los mismos significados en los procesos comunicativos de la lengua meta. Sobre el tema, Anabalón y Vivanco (2014) explican la diferencia entre error y equivocación: error es una desviación de la competencia lingüística por desconocimiento por parte del aprendiente, mientras que equivocación es un desperfecto en el uso de la lengua ligado a la dificultad de pasar del conocimiento teórico a su aplicación. Esta oposición nos permite distinguir con mayor claridad los errores que se cometen en el proceso comunicativo de la lengua extranjera por ignorancia, de las equivocaciones que realizan los aprendientes porque no logran usar lo ya aprendido teóricamente.

En el campo fonético-fonológico, en un proceso normal de aprendizaje, es más frecuente la aparición de equivocaciones porque los aprendientes han recibido de una manera u otra la instrucción por parte del maestro, sin embargo, su oído se encuentra aún en la etapa de ejercitación para lograr escuchar los fonemas específicos de la lengua extranjera, por lo cual es incapaz de distinguir, con fineza, las particularidades de esos sonidos. Sobre este asunto Blanco y Nogueroles (2014) indican que un nivel de competencia fónica insuficiente dificulta, o incluso imposibilita la comunicación tanto a nivel perceptivo como productivo.

Si nuestra atención se orienta en el componente fonológico comprenderemos la importancia de la adquisición de una destreza fonética y fonológica de la lengua que se aprende ya que cada sistema de lengua posee, por un lado, fonemas específicos que deberán ser aprendidos y, por otro, elementos prosódicos particulares que toman un gran peso en los componentes pragmático y sociolingüístico.

\section{PRESENTACIÓN DEL PROYECTO DE INVESTIGACIÓN}

El presente proyecto de indagación toma como punto de partida los resultados de la tesis de maestría de uno de los autores acerca de la autocorrección de la pronunciación de la lengua francesa para futuros maestros de la lengua. En dicho estudio el centro de interés fue la importancia de la corrección fonética del francés a docentes de la lengua en formación para facilitar los procesos comunicativos.

En esta propuesta de estudio se presentan las variaciones fonopragmáticas del español de la región noreste de México que introducen ambigüedad o un cambio de sentido cuando interfieren, bajo forma de atenuación, en los procesos comunicativos en lengua francesa, en hispanohablantes del noreste mexicano, futuros maestros de FLE. Esta indagación se vuelve primordial para fortalecer la enseñanza del francés en esta área ya que cada lengua posee sus propios rasgos fonopragmáticos.

\subsection{Preguntas de investigación}

1. ¿Cuál es la función de los rasgos fonopragmáticos en los procesos comunicativos de una lengua? 
2. ¿Cuál es la incidencia de los rasgos fonopragmáticos del español cuando interfieren en los procesos comunicativos del francés?

3. ¿De qué manera la dimensión cultural del noreste mexicano, presente en el habla del español mediante rasgos fonopragmáticos y actividades de imagen social, genera una interferencia atenuadora en los procesos comunicativos del francés en los aprendientes de esta lengua extranjera?

\subsection{Objetivos de la investigación}

En esta propuesta de investigación los autores se enfocarán en el estudio de los rasgos fonopragmáticos para:

1. Describir su función en los procesos comunicativos de una lengua.

2. Mostrar su incidencia en los procesos comunicativos del francés.

3. Exponer la manera en que los rasgos culturales del noreste mexicano presentes en el habla del español mediante rasgos fonopragmáticos y actividades de imagen social generan una interferencia atenuadora en los procesos comunicativos del francés en los aprendientes de esta lengua extranjera.

\subsection{Hipótesis de partida}

Se enuncia la hipótesis de esta indagación con base en los resultados de una investigación previa (Valdez, 2013), en la cual encontramos que la interferencia atenuadora de los elementos prosódicos en lengua francesa ocurre en la mayoría de los casos en función del género y del origen socio-cultural del hablante. Esta hipótesis se sustenta en el supuesto de que en el proceso comunicativo existen actividades de imagen social que se presentan bajo forma de una atenuación por ideología y prejuicios culturales.

\section{ANTECEDENTES Y MARCO TEÓRICO}

\subsection{Importancia de los elementos fonológicos en el aprendizaje y enseñanza de una lengua extranjera}

En este apartado abordaremos algunos aspectos que nos permitan entender la importancia del estudio de los elementos fonológicos de una lengua extranjera. Rolland (2011) define a la fonología como la encargada de dar significado al sonido, y en este sentido, se encuentra íntimamente ligada a la fonética.

Luchini y García (2015) afirman que la pronunciación juega un rol fundamental en la comunicación. Es por esta razón que Orduz (2012) se interroga acerca de la influencia de la fonología de la lengua materna en los procesos comunicativos del inglés como segunda lengua o extranjera.

En el contexto de los estudios enfocados en la corrección de la pronunciación, Jiménez y Rosales (2013) consideran importante la práctica y la corrección de la entonación en el aula mediante el desarrollo de las técnicas más óptimas para el dominio de la competencia fonológica, en función de las capacidades de los aprendientes para que las pongan en uso y logren así una expresión oral adecuada con el sistema fonológico de la lengua meta. 
Algunas investigaciones relacionadas con la corrección fonológica como la realizada por Luchini y García (2015) se enfocan en indagar acerca del grado del acento extranjero y su fluidez en la pronunciación de la lengua extranjera. Los autores convencidos de la necesidad de buscar técnicas de corrección en el plano fonológico, se dieron a la tarea de contrastar dos metodologías correctivas para determinar cuál es la más eficaz en el plano fonológico. La existencia de investigaciones a nivel fonológico deja entrever que no se trata de la búsqueda de una pronunciación melódicamente perfecta. Se trata más bien de la búsqueda de una pronunciación comprensible, fonética y fonológicamente, para el grupo lingüístico de la lengua meta.

Algunos autores como Jiménez y Rosales (2013) consideran que el docente dedicado al trabajo de fonética correctiva debe realizar, al mismo tiempo, un trabajo de corrección fonológica. De cierta manera, la corrección prosódica debería ser indisociable del trabajo de corrección fonética. Fernández (2014) afirma incluso que el primer medio de comunicación que el ser humano utiliza es mediante los rasgos prosódicos, de tal manera que la dimensión prosódica debería ser lo primero en corregir de acuerdo con el autor.

Los diferentes autores dedicados al estudio de la fonología concuerdan en afirmar que es fundamental continuar generando reflexiones e investigaciones sobre este campo, muy a menudo descuidado, puesto que se considera que la corrección fonética bastaría. Sin embargo, es imposible separar estas dos áreas de la lingüística que confluyen a facilitar los procesos comunicativos orales en cada una de las lenguas.

\subsection{Importancia de la competencia fonológica de una lengua extranjera}

Antes de profundizar en los rasgos fonopragmáticos, entendamos primeramente que uno de los problemas más comunes al aprender una lengua extranjera se encuentra en el campo fonético-fonológico, como afirman Anabalón y Vivanco (2014). Se trata de los sonidos cuya singularidad introduce cambio de significado en la lengua extranjera pero que, en la lengua materna son sonidos alofónicos. ¿De dónde viene esta dificultad? En el aprendizaje de lenguas extranjeras resulta evidente el atasco que muchos aprendientes experimentan en el plano fonético-fonológico. La problemática identificada por Blanco y Nogueroles (2014) se sitúa a nivel de la falta de dominio de la competencia fonético-fonológica de la L2, que es una pieza fundamental de la competencia comunicativa puesto que esta carencia repercute directamente en la percepción y la expresión oral en la lengua meta.

Ribera (2013) y Gajos (2015) especifican que la impericia en la competencia fonéticofonológica de L2 consiste en primer lugar en la percepción equívoca de los fonemas específicos de la lengua meta asociándolos con los sonidos más parecidos existentes en L1.

Lo anterior nos deja entender que la dificultad perceptiva estriba en el hecho de que cada sonido de L2, que es inexistente en L1, se trata en realidad de una configuración articulatoria inexistente en L2 que se vuelve casi imperceptible o difícil de asimilar auditivamente por el aprendiente. Y puesto que la percepción es errónea, habrá forzosamente una repercusión en la pronunciación. En efecto, para Blanco y Nogueroles (2014) estas dificultades en la percepción traen consigo la aparición de las dificultades de pronunciación de la lengua meta.

Aunque el punto de partida en la dificultad para adquirir la competencia fonético-fonológica se sitúa a nivel de la percepción, Anabalón y Vivanco (2014), enfocan su estudio en los efectos del fenómeno perceptivo, es decir en la detección de los errores típicos en la pronun- 
ciación de L2. Los autores dejan en claro a través de su investigación que un trabajo teórico práctico en indispensable para remediar las dificultades que se presentan en este campo. En efecto, el estudio teórico de las particularidades fonéticas y fonológicas de L2, ayudan al aprendiente a asimilar con mayor facilidad los aspectos específicos de la pronunciación de L2, y a buscar en el uso, realizar una pronunciación diferenciada de la lengua materna o de otra lengua extranjera aprendida con anterioridad.

Aunque el fenómeno perceptivo se encuentra en la raíz del problema para la dificultad en adquirir la competencia fonético-fonológica de la lengua meta, estudios en el campo de la pronunciación, como el que realizaron Anabalón y Vivanco (2014) son necesarios para determinar con exactitud los tipos de errores que se presentan en la pronunciación y así, establecer los puntos específicos a trabajar en el aula en el plano perceptivo.

\subsection{Consideraciones diversas para el desarrollo de la competencia fonético-fonológica}

Existen investigaciones como la de Gajos (2015) realizada con polacos aprendiendo francés como lengua extranjera, que dejan de manifiesto la existencia de dificultades en la adquisición de la competencia fonético-fonológica por parte de los aprendientes. Uno de los problemas principales que subraya Gajos (2015) es la dificultad por parte de los polacos para discriminar o producir los sonidos vocálicos específicos de la lengua francesa. La mayor cantidad de sonidos vocálicos presentes en el sistema fonético del francés, con sus dieciséis vocales clásicas y tres semivocales, vuelve difícil el trabajo de asimilación por parte de los polacos, quienes tienen solamente ocho sonidos vocálicos en el sistema fonético de su lengua materna.

Se podría afirmar, de acuerdo con el autor, que el aprendiente polaco cuando oye hablar la lengua francesa emplea involuntariamente para el análisis de lo que oye el sistema fonológico de la lengua polaca. Este problema no radica forzosamente en la incapacidad por parte de los aprendientes polacos en pronunciar un fonema determinado de la lengua meta sino en percibir con error dicho fonema.

En este estudio proponemos que cada grupo lingüístico posee sus propias dificultades en el aprendizaje de una lengua extranjera en función del grado de similitud o de contraste que exista entre el sistema fonético de L1 y de L2. El gran interrogante en el plano didáctico consiste en saber de qué manera y en qué momento dar solución a las dificultades que se presentan en la adquisición de la competencia fonético-fonológica de la lengua meta por parte de los diferentes grupos lingüísticos.

En este orden de ideas, la investigación de Campillos (2013) realizada con diversas agrupaciones lingüísticas aprendiendo el español como lengua extranjera con el objetivo de determinar las dificultades específicas de cada grupo al aprender esta lengua, deja de manifiesto que exploraciones específicas a cada comunidad lingüística deben ser realizadas para determinar las dificultades que se presentan, en particular, en la adquisición de la competencia fonético-fonológica, tanto en función de la lengua meta que se aprende, como de la lengua materna a partir de la cual se lleva a cabo el aprendizaje.

Este tipo de estudios acerca de las dificultades fonéticas en L2 no deberían reducirse solamente al examen de las variaciones específicas ligadas al idioma a partir del cual se aprende la L2. La diversidad geográfica y regional también pueden afectar o favorecer el aprendizaje de los fonemas específicos de la lengua meta. 
Por ejemplo, la investigación de Herrero y Andión (2011) se llevó a cabo con aprendientes de español procedentes de dos grupos con variaciones dialectales en lengua materna. Esta indagación permite entender que las dificultades para aprender los rasgos fonéticos de una lengua extranjera no serán las mismas para aprendientes que, aunque posean una misma lengua materna provengan de diferentes lugares geográficos o regionales.

Por citar un ejemplo, las dificultades para aprender determinada lengua extranjera no serán las mismas para un argentino, un chileno y un mexicano debido a las variaciones de pronunciación que existen entre estos distintos grupos que, aunque comparten el español como lengua materna, la diversidad geográfica y regional han introducido en ellas variaciones a nivel fonético y lexical principalmente produciendo efectos favorables o desfavorables en el proceso de aprendizaje de la lengua meta.

Incluso dentro de un mismo país podrían darse este tipo de variaciones como es el caso de la pronunciación aspirada de la /s/ por hablantes de la región de Tabasco (México) en contraste con la manera en que es pronunciada por la mayoría de los hispanohablantes del resto de la población mexicana. Ellos tendrían probablemente mayor dificultad para distinguir la /s/ de la /z/. En la investigación previa realizada por Valdez (2013) con hispanohablantes del noreste mexicano aprendientes del francés, se obtuvo información que deja entrever las dificultades de los participantes para identificar el sonido /3/ por lo cual usan la yod /j/ en su lugar. Eso ocurre por ser el fonema más parecido que existe en su propio sistema de lengua dialectal.

Ahora bien, acerca de esta variabilidad fonética entre L1 y L2, Derakhshan y Karimi (2015) consideran que nuestra atención no solo debe ponerse sobre las diferencias entre ambos sistemas fonéticos de lengua. Los aspectos similares pueden coadyuvar al aprendizaje efectivo con mayor rapidez gracias a esos conocimientos previos que el aprendiente posee de su lengua materna.

Debido a lo anteriormente señalado, la influencia de la lengua materna no hay que considerarla ineludiblemente como negativa. Anabalón y Vivanco (2014) destacan incluso la existencia de una competencia interlengua que no es ni la competencia de la lengua materna ni la competencia de la lengua meta. Es un estado intermedio en donde el aprendiente se sirve de aspectos de la lengua materna y de la lengua meta, ambos confluyendo al logro del proceso comunicativo de la lengua extranjera.

Esta perspectiva abre un vasto campo en la investigación en didáctica de lenguas puesto que numerosas instituciones educativas en México han considerado que la enseñanza de lenguas debe realizarse únicamente en la lengua meta sin hacer intervenir la lengua materna del aprendiente. Habría que interrogarse de qué manera, en contextos educativos bilingües, la intervención de L1 puede ser útil en el aprendizaje de L2. El cuestionamiento permite entrever la gran labor que queda aún por hacer por estudiosos del área para poner estos conocimientos al servicio de docentes y didactas de lenguas, con el propósito de buscar nuevas alternativas metodológicas que faciliten el desarrollo de la competencia fonético-fonológica y coadyuvar así a un aprendizaje integral de una segunda lengua o extranjera.

\subsection{Acerca de los rasgos prosódicos al aprender una lengua extranjera}

Uno de los componentes lingüísticos indispensables en la competencia comunicativa es el fonológico que, de manera última en cuanto a la expresión se refiere, permite aportar 
precisiones de sentido mediante las variaciones prosódicas. Ahora bien, para entender la importancia de la enseñanza de la prosodia, es conveniente hacer la distinción entre la función lingüística y supralingüística de los elementos prosódicos propuesta por Intravaia (2000). En efecto, en la función lingüística nuestra atención se centra en las dificultades de comprensión en la comunicación ligadas al valor semántico y sintáctico, mientras que en la función supralingüística se tendrán en cuenta los elementos suprasegmentales para introducir una variabilidad de matices de sentido en lo que se quiere comunicar haciendo uso de rasgos de expresividad y de recursos discursivos específicos de la lengua de comunicación.

Para este estudio habría que interrogarnos primeramente sobre la importancia de la incorporación de la dimensión prosódica de una lengua en su enseñanza. Para responder a este cuestionamiento, hay que subrayar que, por una parte, las funciones lingüísticas y supralingüísticas de las que hablan autores como Intravaia (2000) y Rolland (2011) se propone el carácter necesario de su enseñanza en las clases de lengua debido a su repercusión en la variación de significado y matices de sentido que ocurren en los procesos comunicativos y, por otra parte, de acuerdo con el MCER (Conseil de l'Europe 2001) se concibe una progresión en el desarrollo de la capacidad que tiene un estudiante para utilizar los recursos lingüísticos y supralingüísticos hasta la adquisición de un nivel elevado en el C2, donde se domina ampliamente un completo repertorio de elementos lingüísticos para formular pensamientos con precisión, poner énfasis, diferenciar y eliminar la ambigüedad. En este nivel los estudiantes no manifiestan ninguna limitación sobre lo que quieren decir.

Así pues, un primer aspecto que se profundizará en la presente investigación es describir los códigos prosódicos de la lengua meta para aportar al conocimiento, diseñar estrategias didácticas y promover el dominio de las variaciones que introducen un cambio de sentido y que son específicas a la comunicación en francés de los hablantes del noreste mexicano.

En este sentido, se propone partir de la distinción entre la función lingüística y supralingüística de la prosodia (Intravaia 2000, Rolland 2011). Intravaia (2000) afirma que los elementos rítmico-melódicos tienen una importancia fundamental en todas las lenguas porque ellos juegan primeramente un rol semántico. Siguiendo este orden de ideas, la primera función lingüística en la que se piensa es la función distintiva en las oposiciones modales no marcadas gramaticalmente. La melodía sirve entonces para determinar el modo del enunciado. Por ejemplo, el enunciado Vous m'attendez (Usted me espera) podría ser pronunciado de manera diferente para introducir variaciones de sentido y ser considerado como una declaración, una interrogación o bien ser dicho de manera imperativa. Sobre esta situación Rolland (2011) afirma que en un enunciado afirmativo la sílaba tónica es descendiente mientras que en un interrogativo es ascendiente.

Por otra parte, Intravaia (2000) subraya que en francés utilizamos un acento que denominamos "de insistencia" el cual puede tener en ciertos contextos una función distintiva cuando el punto culminante da al enunciado un contenido diferente. Por ejemplo, el enunciado C'est vrai ce que tu racontes (Es verdad lo que cuentas) puede expresar que estamos reafirmando la veracidad de lo expresado por el interlocutor. Pero también podría significar que no estamos seguros de la veracidad de la enunciación del interlocutor y queremos con este enunciado confirmarla.

La segunda función de la prosodia en los procesos comunicativos de una lengua es la supralingüística. Sobre esta función Intravaia (2000) afirma que los recursos discursivos de 
cada lengua son diversos y permiten expresar toda clase de estados psíquicos como la satisfacción, descontento, desprecio, decepción, enojo, entre otros. Estos aspectos expresivos y emocionales se manifiestan de manera diferente en cada sistema de lengua y representan la función supralingüística de la prosodia en la comunicación.

\subsection{Sobre la función supralingüística de la prosodia y la corrección fonopragmática de una lengua extranjera}

Con respecto a las implicaciones de la función supralingüística de la prosodia en los procesos comunicativos (Intravaia 2000), concordamos con lo enunciado por Hidalgo (2009) cuando afirma que los rasgos prosódicos son fundamentales a la hora de delimitar la modalidad enunciativa puesto que la entonación contribuye en los procesos comunicativos modificando el significado o sentido de lo que se expresa. Sin embargo, en la comunicación la prosodia no es el único recurso con el que se cuenta para la comprensión de la enunciación puesto que la pragmática, aporta elementos discursivos para su interpretación. Es por esta razón que abordamos el presente estudio desde la fonopragmática porque en esta disciplina postulada por Hidalgo (2009) converge el estudio de los elementos fonéticos y/o fonológicos y de la pragmática. En efecto, esta concurrencia se debe a que la prosodia, por una parte, expresa "la intención última del hablante" (Hidalgo 2011: 18) y, por otra parte, sugiere una interpretación pragmática determinada (Hidalgo 2009: 173).

Podríamos decir que la prosodia es utilizada como un recurso discursivo. Por ejemplo, la atenuación es definida por Briz (2006: 7-8) como "una categoría pragmalingüística cuya función consiste en minimizar la fuerza ilocutiva de los actos de habla y el papel de los participantes de la enunciación”. Briz (2005: 51) explica que la atenuación tiene por objetivo buscar "la aceptación del oyente, ya sea de lo dicho y del decir o del propio hablante".

De acuerdo con Hidalgo (2009: 174) "la función de atenuación en el ámbito fónico suprasegmental corresponde esencialmente a la entonación". La prosodia, en este sentido, es un recurso que permite interpretar la enunciación como atenuada o no. Briz (2006) advierte que los recursos discursivos de atenuación o intensificación son identificables sin dificultad por el poder que estos conllevan en sí mismos, pero subraya que estos recursos atenuantes o intensificadores pueden variar según la lengua ya que cada una de ellas establece marcas propias de tales gradaciones.

Ahora bien, ¿de qué manera estos recursos discursivos se presentan en la prosodia para manifestar verbalmente la atenuación o la cortesía? ¿Estos recursos discursivos varían según la lengua? ¿Qué diferencias existen entre el español y el francés en cuanto al sentido que brindan los rasgos fonopragmáticos de estas lenguas? Para responder a estos cuestionamientos, es importante subrayar que cada lengua posee sus propios mecanismos de comunicación que le singularizan, los cuales se encuentran impregnados de elementos socioculturales específicos de la comunidad lingüística de la que se trate.

Sobre este asunto, Venel (2012) afirma que cada lengua se encuentra vinculada a la cultura específica de cada grupo lingüístico. Por su parte, Hoefstede (1991) señala que la pertenencia a una cultura genera en los hablantes de una lengua una especie de programación mental a partir de la cual se desenvuelven e interactúan en sociedad. La gran problemática es que hoy en día, frente a la globalización, la gente interactúa con diferentes grupos lingüísticos que poseen, cada uno de ellos, distintos niveles de programación mental 
en función de sus culturas originarias lo cual repercute, de una manera u otra, en el habla. Por esta razón, aprender una lengua debe implicar también un aprendizaje de los elementos culturales. Hoefstede (1991) menciona que la cultura se manifiesta en una lengua y juega un rol fundamental el contexto regional, étnico, religioso, pero también el género, la edad y la clase social de los hablantes.

Frente a esta situación, Venel (2012) afirma que los aprendientes de lenguas extranjeras deben tener una apertura al aprendizaje de la diversidad cultural existente en los diferentes grupos lingüísticos para alcanzar una comunicación efectiva en cada uno de ellos. Sin embargo, los rasgos culturales que aprendimos desde nuestra infancia pueden frecuentemente eclipsar la apertura a una visión pluricultural a la que nos empuja el aprendizaje de lenguas extranjeras, lo que se refleja en reacciones de tipo etnocéntrico y sociocéntrico provocando que los rasgos fonopragmáticos de la lengua materna se entremezclen en los procesos comunicativos de la lengua extranjera y alteren, frecuentemente, el sentido de lo que se desea comunicar. Nizet y Rigaux (2014) brindan, como una posible explicación a esta situación, la perspectiva de Goffman (1986) quien considera que la sociedad actúa en función de una imagen social que funciona como una máscara mediante la cual trata de controlar las impresiones del interlocutor.

En el estudio piloto realizado con alumnos de la licenciatura en Ciencias del Lenguaje de la Facultad de Filosofía y Letras de la Universidad Autónoma de Nuevo León, al hablar en francés como lengua extranjera, entre los resultados obtenidos de varones se halló que trataron de conservar su imagen ligada a su identidad de género (cuidar la entonación y prosodia "masculina") impregnada de rasgos socioculturales del contexto mexicano, también que, en general, los procesos comunicativos se efectuaron suavizando en lo posible los actos verbales que representaban imposiciones para salvaguardar su imagen frente al interlocutor. Y lo realizaron sin tomar en cuenta los recursos fonopragmáticos específicos de la lengua francesa, por ello su manifestación discursiva se presentó bajo la forma de atenuación, cuando un hablante nativo del francés en las mismas circunstancias, quizá habría utilizado la intensificación como recurso fonopragmático. El hablante nativo de lengua francesa, por lo general, realzaría ciertos elementos de la enunciación para ponderar lo dicho; su manifestación fonopragmática se decodifica en México como intensificación.

En definitiva, proponemos que cada sistema de lengua maneja sus propios recursos discursivos que se manifiestan en variaciones fonopragmáticas que son específicas a cada lengua, y que los aprendientes y profesores deben considerar para comunicarse eficiente y correctamente en la lengua extranjera.

\section{METODOLOGÍA}

Para la construcción de esta propuesta se ha ido desarrollando una ruta de indagación metodológica que ha consistido en: a) exponer en diversas reuniones académicas de docentes de FLE los avances realizados en la investigación; b) impartir talleres con los avances de esta metodología a pares académicos para recibir retroalimentación de los docentes asistentes; c) incorporar estas aportaciones al trabajo con los alumnos de licenciatura en Ciencias del Lenguaje de la Facultad de Filosofía y Letras de la UANL; d) verificar contra la teoría y la implementación la operatividad de los hallazgos y e) realizar las adaptaciones correspondientes. 
El siguiente modelo operativo utilizado originalmente para autocorrecciones de tipo fonético (Valdez, 2013) se adopta para la realización de la autocorrección a nivel prosódico:

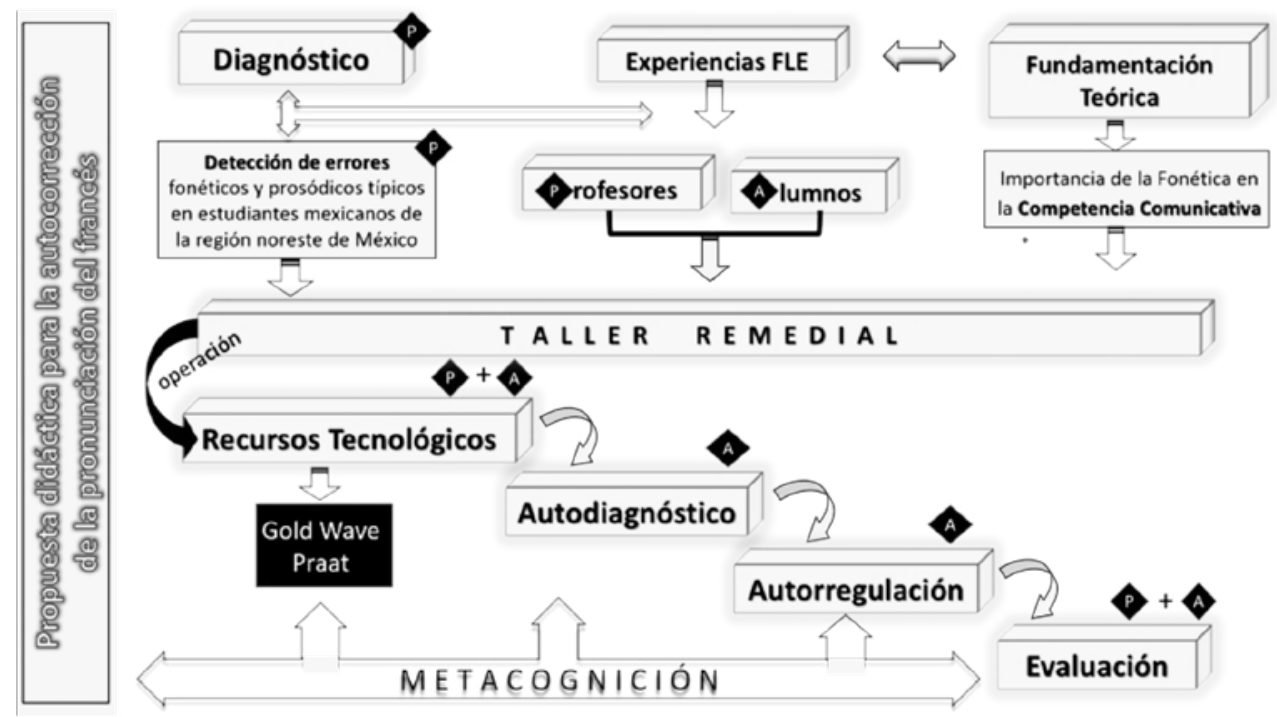

Figura 1. Modelo operativo de investigación.

\subsection{El proceso de autorregulación}

En este estudio proponemos como estrategia para conocer y asimilar progresivamente los rasgos fonopragmáticos de la lengua francesa no solamente la retroalimentación que el profesor pueda aportar, sino también un trabajo de autorregulación mediante el cual los aprendientes logren descubrir por ellos mismos las variaciones fonopragmáticas que existen entre su lengua materna y la lengua meta.

Retomamos aquí la definición de autorregulación propuesta por Panadero y Alonso-Tapia (2014: 450-451) quienes afirman que este proceso se trata del control que el aprendiente realiza sobre sus acciones, pensamientos, emociones y motivación mediante estrategias de aprendizaje individuales que le permiten alcanzar eficazmente las metas propuestas.

Esta eficacia dependerá del grado de compromiso y motivación que el estudiante imprima en su aprendizaje. En este sentido consideramos que la autorregulación es una pieza clave en esta área del conocimiento, puesto que a nivel de la pronunciación de una lengua extranjera los aprendientes podrán profundizar en este proceso de aprendizaje tanto como lo deseen. Concordamos con García-Jiménez (2015: 17) en su definición de autorregulación quien la concibe como "un proceso por el que los estudiantes deciden lo que quieren aprender y con qué nivel de profundidad y después intentan hacer un seguimiento de lo aprendido, controlando y modificando, cuando es necesario, sus estrategias de aprendizaje". Este tipo de aprendizaje autorregulado es el que proponemos para este estudio.

Debido al objetivo didáctico de la presente investigación se recurrirá a un corpus inducido. En efecto, se busca que el futuro docente de FLE detecte por él mismo las va- 
riaciones que existen entre los elementos prosódicos de ambas lenguas y, a partir de este contraste, sea capaz de autocorregirse y realizar un acercamiento, en el uso, a los rasgos fonopragmáticos del francés.

Los estudiantes grabarán diversas situaciones comunicativas propuestas por género en las cuales deben pronunciar poniendo cuidado, en un primer momento, en los elementos fonéticos de la lengua francesa. Posteriormente deberán integrar los rasgos prosódicos pertinentes de acuerdo al contexto del proceso comunicativo.

Para este trabajo de autocorrección se ha diseñado un instrumento. El estudiante deberá usar la Tabla 1 para contrastar y registrar rasgos de la pronunciación del nativo y la suya con el objetivo de detectar las variaciones presentadas en el proceso comunicativo:

\begin{tabular}{|c|c|c|c|c|}
\hline $\mathrm{N}^{\circ}$ práctica: & Nombre & & & \\
\hline \multicolumn{5}{|c|}{ Grabación del alumno SIN escucha previa } \\
\hline \multicolumn{5}{|l|}{ Frase: } \\
\hline & \multicolumn{4}{|c|}{ Descripción del error: } \\
\hline & Error 1 & Error 2 & Error 3 & Error 4 \\
\hline \multicolumn{5}{|l|}{$\begin{array}{l}\text { Pronunciación de } \\
\text { fonemas }\end{array}$} \\
\hline \multicolumn{5}{|l|}{ Acento fijo } \\
\hline \multicolumn{5}{|l|}{$\begin{array}{l}\text { Acento de } \\
\text { insistencia }\end{array}$} \\
\hline \multicolumn{5}{|l|}{ Curva melódica } \\
\hline \multicolumn{5}{|c|}{ Grabación del alumno posterior a la primera escucha } \\
\hline & \multicolumn{4}{|c|}{ Descripción del error: } \\
\hline & Error 1 & Error 2 & Error 3 & Error 4 \\
\hline \multicolumn{5}{|l|}{$\begin{array}{l}\text { Pronunciación de } \\
\text { fonemas }\end{array}$} \\
\hline \multicolumn{5}{|l|}{ Acento fijo } \\
\hline \multicolumn{5}{|l|}{$\begin{array}{l}\text { Acento de } \\
\text { insistencia }\end{array}$} \\
\hline \multirow{2}{*}{\multicolumn{5}{|c|}{ rabación del alumno posterior a la }} \\
\hline & & & & \\
\hline & \multicolumn{4}{|c|}{ Descripción del error: } \\
\hline & Error 1 & Error 2 & Error 3 & Error 4 \\
\hline \multicolumn{5}{|l|}{$\begin{array}{l}\text { Pronunciación de } \\
\text { fonemas }\end{array}$} \\
\hline \multicolumn{5}{|l|}{ Acento fijo } \\
\hline \multicolumn{5}{|l|}{$\begin{array}{l}\text { Acento de } \\
\text { insistencia }\end{array}$} \\
\hline \multicolumn{5}{|l|}{ Curva melódica } \\
\hline \multicolumn{5}{|c|}{ Grabación del alumno posterior a la tercera escucha } \\
\hline & \multicolumn{4}{|c|}{ Descripción del error: } \\
\hline & Error 1 & Error 2 & Error 3 & Error 4 \\
\hline $\begin{array}{l}\text { Pronunciación de } \\
\text { fonemas }\end{array}$ & & & & \\
\hline Acento fijo & & & & \\
\hline $\begin{array}{l}\text { Acento de } \\
\text { insistencia }\end{array}$ & & & & \\
\hline Curva melódica & & & & \\
\hline
\end{tabular}

Tabla 1. Tabla de contrastación para llevar a cabo el proceso de autorregulación. 
El proceso de la autorregulación se ve facilitado por el instrumento de contrastación que permite a los estudiantes llevar un control progresivo y autónomo de los errores que cometen en su pronunciación, así como la oportunidad de irlos mejorando a través de las diferentes escuchas, en diversos momentos en los que se deberá trabajar de manera específica cada uno de los errores encontrados para volverse a grabar una vez que se considere que ya se haya logrado pronunciar con corrección.

Los pasos del procedimiento que se realiza son los siguientes:

1. El estudiante graba con su voz en GoldWave las frases sin escucha previa del nativo tres veces. La primera es la pronunciación de la frase tal cual se considera deba ser proferida según sus conocimientos sobre la articulación de la lengua. El segundo audio deberá reflejar el mejor esfuerzo de autocorrección fonética. El tercer audio se enfocará en la autocorrección prosódica sin descuidar los elementos fonéticos.

2. Del tercer audio donde se hizo una autocorrección fonética-prosódica se procede a obtener mediante el PRAAT el espectrograma y el oscilograma con la curva melódica de cada una de las frases.

3. Obtener el espectrograma y el oscilograma con la curva melódica de las frases pronunciadas por los nativos en el PRAAT.

4. Contrastar los gráficos del nativo con los obtenidos por el estudiante y tomar nota en la hoja de contrastación de las variaciones encontradas. Elaborar una tabla de contrastación, por frase.

5. Realizar una primera escucha de la frase pronunciada por el nativo y repetir en voz alta varias veces tratando de imitarlo, atendiendo las variaciones que fueron plasmadas en la tabla de contrastación.

6. Una vez que se estime que se haya logrado autocorregir las variaciones descritas en la tabla de contrastación, se procede a grabarse en GoldWave y a editar el archivo de voz obtenido (esta grabación constituye el primer esfuerzo de autocorrección posterior a la escucha del nativo).

7. Se obtiene en el PRAAT el espectrograma y el oscilograma correspondiente a este primer esfuerzo posterior a la escucha del nativo.

8. Se procede a un segundo contraste con los gráficos del nativo y se toma nota en la hoja de contrastación de las variantes detectadas.

9. Realizar una segunda escucha de la frase pronunciada por el nativo y repetir en voz alta varias veces tratando de imitarlo, poniendo especial atención en las diferencias que persisten y que se anotaron en la tabla de contrastación.

10. Cuando se considere que se haya logrado autocorregir las variaciones descritas en el instrumento de contrastación, se procede a grabarse en GoldWave y a editar el archivo de voz obtenido (esta grabación constituye el segundo esfuerzo de autocorrección posterior a la escucha del nativo).

11. Se obtiene en el PRAAT el espectrograma y el oscilograma correspondiente a este segundo esfuerzo.

12. Se procede a un tercer contraste con los gráficos del nativo y se toma nota en la tabla de contrastación de las variaciones que aún persisten.

13. Realizar una tercera escucha de la frase pronunciada por el nativo y repetir en voz alta varias veces tratando de imitarlo. Se atienden especialmente las alteraciones que permanecen y que fueron reportadas en la tabla de contrastación. 
14. Cuando se considere que se haya logrado autocorregir las variaciones descritas en la tabla de contrastación, se procede a grabarse en GoldWave y a editar el archivo de voz obtenido (esta grabación constituye el tercer esfuerzo de autocorrección posterior a la escucha del nativo).

La grabación correspondiente al tercer esfuerzo representa la fase de perfeccionamiento descrita en la tabla de evaluación apreciativa. Por lo tanto, se recomienda que este procedimiento se repita para el tercer esfuerzo cuantas veces se considere necesario hasta mejorar la pronunciación de la frase tanto como se desee perfeccionar y obtener la grabación que más se apegue a la pronunciación del nativo.

Para realizar este trabajo de autocorrección es importante llevar a cabo la enmienda mediante el método de progresión y regresión propuesto por Intravaia (2000). Por otra parte, es importante entender la intención de comunicación del nativo, lo que desea comunicar a través de cada una de las situaciones específicas, para comprender la función comunicativa que ejercen los rasgos prosódicos y buscar así apropiarse de ellos a través de este método contrastivo.

\section{CONCLUSIÓN}

Un aspecto que es vital entender al momento de enseñar una lengua extranjera es tomar conciencia de que las interferencias y dificultades fonéticas en la pronunciación de una lengua meta no son las mismas para los diferentes grupos lingüísticos, por lo que es necesario reconocer los errores fonéticos y prosódicos típicos de los aprendientes de una lengua meta, considerados desde una perspectiva geográfica y sociocultural.

Por citar un ejemplo, los hispanohablantes del noreste mexicano al aprender el francés como lengua extranjera tendrán como dificultades específicas la discriminación /b/-/v/, discriminación /s/-/z/, discriminación /u/-/y/, entre otras. Asimismo, se propone que la dificultad en la realización de grupos melódicos y otros aspectos sin duda presentarían variantes si se trata de aprendientes argentinos, o del centro o sur de México, debido a que la misma diversidad dialectológica y geográfica involucra matices en la producción fónica y prosódica.

Con esta investigación queremos hacer aportación sobre la incidencia de los rasgos supralingüísticos, que, según nuestra hipótesis, juegan un papel importante en la realización e interpretación del mensaje expresado en lengua francesa por los aprendientes mexicanos del noreste del país, debido a los matices atenuadores introducidos por el condicionamiento semiótico-cultural del hablante en la expresión.

De esta manera, el considerar la interpretación del lenguaje y elementos supralingüísticos para la enseñanza, se convierte en un aspecto fundamental para la posterior adquisición de la competencia comunicativa de esta lengua extranjera.

\section{PERSPECTIVAS}

Este proyecto de investigación desea brindar elementos de reflexión a docentes y estudiosos del área acerca de las estrategias didácticas que deberían implementarse para la enseñanza de los elementos prosódicos de la lengua francesa en el contexto mexicano. En efecto, con esta indagación se pretende realizar aportación para mostrar la manera en 
que la función supralingüística de la prosodia en la comunicación aporta variaciones de sentido en función de la lengua, pero también del género y de la cultura del hablante.

Como se sabe, hay una variación melódica entre el hablar de un hombre y el de una mujer. Pero, en el aprendizaje de la lengua francesa ¿de qué manera el factor cultural de género del aprendiente hispanohablante del noreste de México repercute en los elementos prosódicos normativos de la lengua francesa y, por ende, interfiere en los procesos comunicativos de la lengua meta? He aquí los cuestionamientos que guiarán las siguientes fases de esta indagación.

\section{Referencias bibliográficas}

Anabalón, R. y C. Vivanco (2014). “Análisis de desviaciones en la pronunciación de segmentos consonánticos producidas por hablantes de español de chile aprendientes de inglés", Lenguas modernas, 43, pp. 11-25.

Blanco, A. y M. F., Nogueroles (2014). "Errores fónicos de producción en español/L2. Una propuesta de categorización”, Revista Española de Lingüistica Aplicada, 27 (2), pp. 255-274.

Briz, A. (2005). "Eficacia, imagen social e imagen de cortesía. Naturaleza de la estrategia atenuadora en la conversación coloquial española". En Bravo, D. (ed.). Estudios de la (des)cortesía en español. Categorías conceptuales y aplicaciones a corpus orales y escritos. Estocolmo-Buenos Aires: Dunken, pp. 51-89.

Briz, A. (2006). "Para un análisis semántico, pragmático y sociopragmático de la cortesía atenuadora en España y América", LEA, 28 (2), pp. 1-41.

Campillos, L. (2013). "Análisis de la producción y de errores en un corpus oral de español como lengua extranjera", Revista Iberoamericana de Lingüistica, 8, pp. 5-43.

Chiss, J. L., J. Filliolet y D. Maingueneau (2013). Introduction à la linguistique française. Tome: notions fondamentales, phonétique, lexique. Paris : Hachette.

Conseil de l'Europe (2001). Cadre européen commun de référence pour les langues. Apprendre, enseigner, évaluer. Paris : Didier.

Derakhshan, A. y E. Karimi (2015). The interference of first language and second language acquisition. Theory \& Practice in Language Studies, 5 (10), pp. 2112-2117.

Fernández, M. Á. (2014). La adquisición de las relaciones entre prosodia e intención comunicativa: primeras asociaciones entre forma y función. Lexis. Revista de Lingüistica y Literatura, 38 (1), pp. 5-33.

Gajos, M. (2015). "Les étudiants polonophones face aux voyelles nasales françaises. (French)", Neofilolog, 44 (1), pp. 21-37.

García-Jiménez, E. (2015). "La evaluación del aprendizaje: de la retroalimentación a la autorregulación. El papel de las tecnologías. (Spanish)”, Relieve: Revista Electrónica de Investigación y Evaluación Educativa, 21 (2), pp. 1-24.

Goffman, E. (1986). Frame analysis, an essay on the organization of experience. Northeastern, Univ. Press.

Herrero, A., y M.A. Andión (2011). "La enseñanza de la fonética del español a hablantes de escocia e Irlanda del norte. Estudio de casos", Círculo de Lingüística Aplicada a la Comunicación, 47, pp. 28-64.

Herrero, A. y M.A. Andión (2012). "La fonética de aprendices de español del sur y del norte de Inglaterra. Consideraciones acerca de su enseñanza", Revista Española de Lingüística Aplicada, 25, pp. 45-68.

Hidalgo, A. (2009). "Modalización (des)cortés y prosodia: estado de la cuestión en el ámbito hispánico", Boletín de Filología, 44 (1), pp. 161-195.

Hidalgo, A. (2011). "La investigación de la entonación coloquial: hacia un estado de la cuestión en el ámbito hispánico", Oralia, 14, pp. 15-45. 
Hoefstede, G. (1991). Cultures and Organization: Software of the Mind. New York: McGraw-Hill Book.

Intravaia, P. (2000). Formation des professeurs de langue en phonétique corrective. Le système verbotonal. París : Didier Érudition.

Jiménez, M. y M. Rosales (2013). "Propuesta práctica de entonación en el aula de ELE", Revista Nebrija de Lingüística Aplicada a la Enseñanza de Lenguas, 13, pp. 589-595.

Luchini, P. L. y M.A. García (2015). "Sobre el grado de acento extranjero y fluidez en la clase de pronunciación inglesa: un estudio evaluativo", Organon, 30 (58), pp. 193-213.

Nizet, J. y N. Rigaux (2014). La sociologie de Erving Goffman. Paris: La Découverte.

Orduz, Y. (2012). "La transferencia de la fonología de la lengua materna en la adquisición del inglés como lengua extranjera", Revista Entornos, 25, pp. 91-103.

Panadero, E. y J. Alonso-Tapia (2014). “¿Cómo autorregulan nuestros alumnos? Revisión del modelo cíclico de Zimmerman sobre autorregulación del aprendizaje”, Anales de Psicología, 30 (2), pp. 450-452. DOI: 10.6018/analesps.30.2.167221.

Ribera, A.I. (2013). "La didáctica de la pronunciación de ELE, en contexto francés, a través de la asociación de sonidos, gestos y ritmos", Revista Nebrija de Lingüística Aplicada a la Enseñanza de Lenguas, 13, pp. 989-998.

Rolland, Y. (2011). Apprendre à prononcer : Quels paradigmes en didactique des langues ? Paris: Éditions Belin.

Trajkova, M. (2014). "Intégration des technologies de l'information et de la communication dans l'enseignement du français langue étrangère : un exemple de cours de Phonétique du français. (French)", Synergies Roumanie, 9, pp. 35-42.

Vaissière, J. (2011). La phonétique. Paris : Presses Universitaires de France.

Valdez, O. (2013). "Importancia de la corrección fonética en la competencia comunicativa de la lengua francesa", Cathedra, 18, pp. 29-34.

Venel, M.-D. (2012). "La culture de 1'Autre : Un miroir?”, Synergies Mexique, 2, pp. 71-82.

Yang, Y. (2015). "De la perception auditive à la communication langagière : approche interactive en compréhension et expression orales pour l'enseignement du français. (French)", Synergies Chine, 10, pp. 77-87. 
